\title{
Research Article \\ Oscillatory Behavior in Linear Difference Equations under Unmodeled Dynamics and Parametrical Errors
}

\author{
M. De la Sen \\ Received 5 May 2006; Accepted 22 March 2007 \\ Recommended by Jan Awrejcewicz
}

This paper investigates the presence of oscillating solutions in time-varying difference equations even in the case when there exist parametrical errors (i.e., errors in the sequences defining their coefficients) and/or unmodeled dynamics, namely, the current order is unknown and greater than the nominal known order. The formulation is related to the concepts of conjugacy, disconjugacy, positivity, and generalized zeros and general conditions of oscillation are obtained both over particular intervals and for the whole solution. Some results concerned with the presence of stable oscillations are also presented.

Copyright (c) 2007 M. De la Sen. This is an open access article distributed under the Creative Commons Attribution License, which permits unrestricted use, distribution, and reproduction in any medium, provided the original work is properly cited.

\section{Introduction}

The study of oscillations in discrete systems is very important in practice since the achievement oscillations are a design objective on occasions while sometimes are a serious drawback. An example of the first situation is the design of electronic oscillators in radio, $\mathrm{TV}$, communications, in general, and so forth by generating limit cycles in the solutions, that is, asymptotically periodic steady-state solutions which are independent of the initial conditions and which are due to the presence of certain nonlinearities in the dynamic systems like, for instance, saturations. However, very often, such a steady-state solution is a drawback to be avoided such as in servo-design where the steady-state solution should asymptotically track a constant or ramp-type forcing terms. The term "oscillatory solution" is often taken as a synonymous of "periodic solution" in engineering applications. In a mathematical sense, a solution is periodic if its values are repeated with a period while an oscillatory solution is that which changes its sign after a finite (nonnecessarily constant) time interval, [1-5]. In particular, an oscillation theory for functional differential equations with retarded argument is given in [1] while interval oscillation criteria 
based on the information on a sequence of subintervals are derived in [2]. This paper is devoted to the study of the conditions for the presence of oscillatory solutions (see, e.g., [1-10]) in time-varying linear difference equations and their associate dynamic systems. The main objectives of this paper are to investigate the conditions of oscillatory solutions for time-varying difference equations of arbitrary order by generalizing previous detailed analysis for the second-order case given in detail in [5] linked to the concepts of generalized zeros. The oscillations are characterized as strong if the change of sign takes place in one interval equal to the order of the difference equation or weak if, in general, the sign of change takes places for any interval of arbitrary finite measures. The oscillation is said to be strict if the sign change in the solution takes place at each new sample (a solution value at any discrete-time) of the solution. The joint oscillatory and stable/behaviors of the solutions $[6-8,11,12]$ are investigated. An associate dynamic system is also studied in terms of oscillatory solutions, and so forth. Throughout the paper, special attention is devoted to the case when there are parametrical errors and/or unmodeled dynamics. This happens in maneuvers of missile tracking or in high frequency capacitor tandems.

\section{Linear difference equation with uncertainties and unmodeled dynamics}

2.1. Nominal linear difference equation. Consider the $n_{0}$ th-order nominal linear difference equation

$$
\sum_{j=0}^{n_{0}} \beta_{j}^{0}(k) x^{0}(k+1-j)=0, \quad k \in \mathbb{N}_{n_{0}-1}:=\left\{n_{0}-1, n_{0}, \ldots\right\} \subseteq \mathbb{N}_{0}=\mathbb{N} \cup\{0\}
$$

for all $n_{0} \in \mathbb{N}$ (the set of natural numbers) subject to initial conditions, $x^{0}(i)=x_{i} \in \mathbb{R}$ for $i \in \mathbb{N}_{0} / \mathbb{N}_{n_{0}}$ being the set initial conditions, and $\left\{\beta_{j}^{0}(k)\right\}_{0}^{\infty}$ for $j \in \mathbb{N}_{0} / \mathbb{N}_{n_{0}+1}$ being $\left(n_{0}+1\right)$ sequences of real numbers with $\beta_{0}^{0}(k) \neq 0$, for all $k \in \mathbb{N}_{0}$. The values of the solution of (2.1) are popularly known as "samples" since they are only defined for a running integer (i.e., discrete) integer. For simplicity, the abbreviated notation $[a, a+b]:=\mathbb{N}_{a} / \mathbb{N}_{a+b+1}=$ $\{a, a+1, \ldots, a+b\}$ for any $a, b(>a) \in \mathbb{N}_{0}$ will be used. Equation (2.1) is rewritten as

$$
x^{0}(k+1)=-\sum_{j=1}^{n_{0}} \alpha_{j}^{0}(k) x^{0}(k+1-j)
$$

with $\left\{\alpha_{j}^{0}(k):=\beta_{j}^{0}(k) / \beta_{0}^{0}(k)\right\}_{0}^{\infty}$, for all $j \in\left[1, n_{0}\right]$. A discrete nominal linear time-varying dynamic system of state $z^{0}(k)=\left(x^{0}(k), x^{0}(k-1), \ldots, x^{0}\left(k-n_{0}+1\right)\right)^{T}$ is associated with (2.2) to yield

$$
z^{0}(k+1)=A^{0}(k) z^{0}(k), \quad k \in \mathbb{N}_{n_{0}-1}, \quad z^{0}\left(n_{0}-1\right)=\left(x^{0}\left(n_{0}-1\right), x^{0}\left(n_{0}-2\right), \ldots, x^{0}(0)\right)^{T},
$$

where

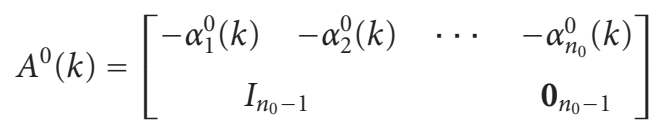


with $I_{j}$ being the $j$ th identity matrix and $\mathbf{0}_{j}=(0,0, \cdots, 0)^{T} \in \mathbb{R}^{j}$. Note that $z^{0}(i) \neq 0$ for all $i \geq k$ and any nonzero $z^{0}(k)$ if and only if $A^{0}(k)$ is nonsingular for all $i \geq k$ which holds if and only if $\alpha_{n_{0}}^{0}(k) \neq 0$. If general, $z^{0}(k+1) \neq 0$ for any combination of values of the coefficients $\alpha_{i}^{0}(k), i \in\left[1, n_{0}\right]$, if and only if $z^{0}(k) \notin \operatorname{Ker}\left(A^{0}(k)\right)$.

2.2. Parametrical errors and unmodeled dynamics. If the order of the difference equation (2.2) is $n>n_{0}$ but it is erroneously described as being of $n_{0}$ th-order and, furthermore, the nominal coefficients are subject to measuring errors or disturbances, that is, $\alpha_{j}^{0} \rightarrow \alpha_{j}, j \in\left[1, n_{0}\right]$, then the nominal (2.2) is replaced with the current $n$ th-order equation,

$$
\begin{aligned}
x(k+1) & =-\sum_{j=1}^{n_{0}} \alpha_{j}(k) x(k+1-j)-\sum_{j=n_{0}+1}^{n} \alpha_{j}(k) x(k+1-j) \\
& =-\sum_{j=1}^{n_{0}} \alpha_{j}(k) x(k+1-j)+\theta_{\mathrm{um}}^{T}(k) \varphi(k)=\tilde{\theta}^{T}(k) \tilde{z}(k) \\
& =\theta^{T}(k) z(k)+\theta_{\mathrm{um}}^{T}(k) \varphi(k)=-\sum_{j=1}^{n_{0}} \alpha_{j}^{0}(k) x(k+1-j)+\eta(k),
\end{aligned}
$$

where

$$
\begin{aligned}
\tilde{\theta}^{T}(k) & =\left(\theta^{T}(k), \theta_{\mathrm{um}}^{T}(k)\right), \quad \tilde{z}^{T}(k)=\left(z^{T}(k), \varphi^{T}(k)\right), \\
\theta^{T}(k) & =\left(-\alpha_{1}(k),-\alpha_{2}(k), \ldots,-\alpha_{n_{0}}(k)\right), \quad \theta^{0^{T}}(k)=\left(-\alpha_{1}^{0}(k),-\alpha_{2}^{0}(k), \ldots,-\alpha_{n_{0}}^{0}(k)\right), \\
\theta_{\mathrm{um}}^{T}(k) & =\left(-\alpha_{n_{0}+1}(k),-\alpha_{n_{0}+2}(k), \ldots,-\alpha_{n}(k)\right), \quad z^{T}(k)=\left(x(k), x(k-1), \ldots, x\left(k+1-n_{0}\right)\right), \\
\varphi^{T}(k) & =\left(x\left(k-n_{0}\right), x\left(k-n_{0}-1\right), \ldots, x(k+1-n)\right)^{T}, \\
\eta(k) & =\left(\theta^{T}-\theta^{0^{T}}\right) z(k)+\theta_{\mathrm{um}}^{T}(k) \varphi(k) \\
& =\sum_{j=1}^{n_{0}}\left(\alpha_{j}^{0}(k)-\alpha_{j}(k)\right) x(k+1-j)+\theta_{\mathrm{um}}^{T}(k) \varphi(k) \\
& =\tilde{\theta}^{T}(k) \tilde{z}(k)-\theta^{T}(k) z(k),
\end{aligned}
$$

where $\theta^{0}(k)$ is the parameter vector of the nominal equation $(2.2), \theta(k)$ is the nominal parameter vector of the current equation $(2.5), \theta(k)-\theta^{0}(k)$ is the parametrical error vector, $\theta_{\mathrm{um}}(k)$ is the parameter vector associated with the unmodeled dynamics, and $\eta(k)$ includes the contributions to the solution of the parametrical errors in the coefficients $\left(\alpha_{j}(k)-\alpha_{j}^{0}(k)\right), j \in\left[1, n_{0}\right]$, of the nominal equation and the unmodeled dynamics, that 
4 Mathematical Problems in Engineering

is, errors associated with an erroneous modeling of the nominal equation order generated by the exceeding coefficients $\alpha_{j}(k), j \in\left[n_{0}+1, n\right]$. An equivalent linear dynamic system to $(2.5)-(2.6)$ is defined by

$$
\widetilde{z}(k+1)=\tilde{A}(k) \tilde{z}(k)
$$

$\forall k \in[n-1, \infty)$ subject to $\widetilde{z}(n-1)=(x(n-1), x(n-2), \ldots, x(1), x(0))^{T}$, where

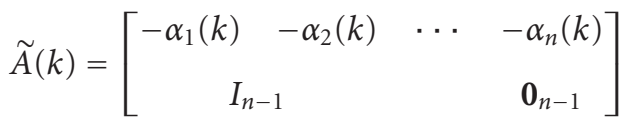

$$
\begin{aligned}
& =\left[\begin{array}{rrrrr}
A(k) & -\alpha_{n_{0}+1}(k) & -\alpha_{n_{0}+2}(k) & \cdots & -\alpha_{n}(k) \\
& I_{n-n_{0}} & & & \mathbf{0}_{n_{0}-1} \\
& & & & \mathbf{0}_{n-n_{0}}
\end{array}\right] \text {, }
\end{aligned}
$$

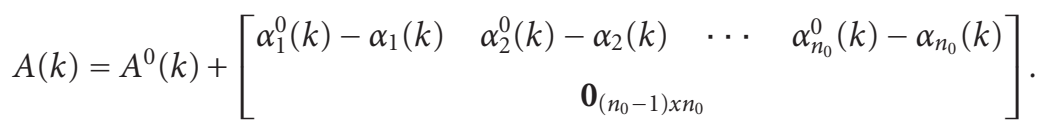

The subsequent result follows from straightforward calculations.

Lemma 2.1. The dynamic system (2.7) is equivalent to

$$
z(k+1)=A(k) z(k)+e_{01} \eta(k)
$$

for $k \geq n_{0}-1$ through (2.6), where $e_{0 i}=(0,0, \ldots 0,1,0, \ldots 0)^{T}$ is the ith Euclidean unity vector in $\mathbb{R}^{n_{0}}$, under initial conditions related by $\tilde{z}(n-1)=\left(x(n-1), x\left(n_{0}+1\right), \ldots, x\left(n_{0}\right)\right.$, $\left.z^{T}\left(n_{0}-1\right)\right)^{T}, z\left(n_{0}-1\right)=\left(x\left(n_{0}-1\right), x\left(n_{0}-2\right), \ldots, x(0)\right)^{T}$.

2.3. Recursive equations. Note from (2.8) that for any $N(k) \in[0, \infty)$,

$$
\begin{aligned}
\tilde{z}(k+N(k)+1) & =\tilde{\mathbf{A}}(k, k+N(k)) \tilde{z}(k)=\left(\prod_{j=k}^{k+N(k)}[\tilde{A}(j)]\right) \tilde{z}(k), \quad \forall k \in[n-1, \infty), \\
z(k+N(k)+1) & =\mathbf{A}(k, k+N(k)) z(k)+\eta(k, k+N(k)) \\
& =\left(\prod_{j=k}^{k+N(k)}[A(j)]\right) z(k)+\sum_{j=k}^{k+N(k)}\left(\prod_{i=j+1}^{k+N(k)}[A(i)]\right) \eta(j), \quad \forall k \in\left[n_{0}-, \infty\right),
\end{aligned}
$$


where

$$
\begin{gathered}
\tilde{\mathbf{A}}(k, k+N(k))=\left(\tilde{\mathbf{A}}_{i j}(k, k+N(k))\right)=\prod_{j=k}^{k+N(k)}[\tilde{A}(j)]=\tilde{\mathbf{A}}(k+N(k)) \tilde{\mathbf{A}}(k, k+N(k)-1), \\
\mathbf{A}(k, k+N(k))=\left(\mathbf{A}_{i j}(k, k+N(k))\right)=\prod_{j=k}^{k+N(k)}[A(j)]=\mathbf{A}(k+N(k)) \mathbf{A}(k, k+N(k)-1), \\
\boldsymbol{\eta}(k, k+N(k))=\sum_{j=k}^{k+N(k)} \prod_{i=j+1}^{k+N(k)}[A(i)] \eta(j)=\mathbf{A}(k+N(k)) \boldsymbol{\eta}(k, k+N(k)-1)+e_{01} \eta(k+N(k)) .
\end{gathered}
$$

The substitution of (2.4) into (2.11) yields in general the following recursive equations for the entries of $\tilde{\mathbf{A}}(k, k+N(k))$ and $\mathbf{A}(k, k+N(k))$ :

$$
\begin{aligned}
\tilde{\mathbf{A}}_{1 j}(k, k+N(k)) & =-\sum_{i=1}^{n} \alpha_{i}(k+N(k)) \tilde{\mathbf{A}}_{i j}(k, k+N(k)-1), \\
\tilde{\mathbf{A}}_{i+1, j}(k, k+N(k)) & =\widetilde{\mathbf{A}}_{i j}(k, k+N(k)-1), \quad \text { for } i \in[1, n-1], j \in[1, n], \\
\mathbf{A}_{1 j}(k, k+N(k)) & =-\sum_{i=j}^{n_{0}} \alpha_{i}(k+N(k)) \mathbf{A}_{i j}(k, k+N(k)-1), \\
\mathbf{A}_{i+1, j}(k, k+N(k)) & =\mathbf{A}_{i j}(k, k+N(k)-1), \quad \text { for } i \in\left[1, n_{0}-1\right], j \in\left[1, n_{0}\right] .
\end{aligned}
$$

\section{Basic definitions and results}

The definitions below classify the oscillations facilitating the subsequent results.

Definitions. Consider intervals of integers $[k, k+N]$ for given $k \in[n, \infty), N \in[n+1, \infty)$, and the solutions of (2.5)-(2.6) under initial conditions $x(i)=x_{i}, i \in[0, n-1]$.

Definition 3.1. A solution is weakly oscillatory (WO) in $[k, k+N]$ if it has at least two sign changes in $[k, k+N]$. If a solution is WO in $[k, k+N]$ for all $k \geq n-1$, then it is globally weakly oscillatory (GWO).

Definition 3.2. A solution is strongly oscillatory (SO) in $[k, k+N]$ if it is WO within each subinterval $[k+i, k+i+n] \subseteq[k, k+N], i \in[0, N-n]$. If a solution is $\mathrm{SO}$ in $[k, k+N]$, for all $k \geq n-1$ and all $N \geq n+1$, then it is globally strongly oscillatory (GSO).

Definition 3.3. A solution is strictly strongly oscillatory (SSO) in $\left[k, k+N_{0}\right]$ for a given integer $N_{0} \geq 1$ if $\delta(k+i, k+i+1):=x(k+i) x(k+i+1)<0$ for all $i \in\left[0, N_{0}-1\right]$. If a solution is SSO in $\left[k, k+N_{0}\right]$ for all $k \geq n-1$ and all $N_{0} \geq 1$, then it is globally strictly strongly oscillatory (GSSO).

Definition 3.4. A solution is oscillatory $(\mathrm{O})$ in $[k, k+N]$, respectively, globally oscillatory $(\mathrm{GO})$ if it is at least WO in $[k, k+N]$, respectively, GWO. 
The trivial solution is neither WO nor SO. Also, an identically zero solution for all $k \geq N_{1}$, some $N_{1} \geq 0$, is not GO but it might be O (resp., WO, SO, SSO) in intervals within $\left[n, N_{1}-1\right]$ if $N_{1} \geq n+1$. Note also that if a solution is SSO for some integers $k \geq n, N_{0} \geq n+1$, then it is also SO and WO in $[k, k+N]$ for each $N \in\left[n+1, N_{0}\right]$. The subsequent result holds.

THeORem 3.5. The following properties hold for any system (2.5)-(2.6) with $n_{0} \geq n \geq 1$ :

(i) if a solution is SSO in $[k, k+N]$ for some integer $N \geq n+1$ (resp., GSSO), then it is $S O$ and $W O$ in $[k, k+N]$ (resp., GSO and GWO);

(ii) if a solution is $S O$ in $[k, k+N]$ for some integer $N \geq n+1$ (resp., GSO), then it is WO in $[k, k+N]$ (resp., GWO);

(iii) if $n_{0}=n=1$, a solution is SSO in $[k, k+1]$ if and only if it is SO in $[k, k+2]$. It is GSSO if and only if it is GSO.

The proof of Theorem 3.5 is immediate and it is omitted. A solution is said to be disconjugate in $[k, k+N]$ when $n=2$, [5], if $\delta(k+i, k+i+1)<0, \delta(k+i+j, k+i+j+$ $1)<0, i \in[0, N-1], j \in[1, N-i]$ provided that $x(k+i-1)=x(k+i+j-1)=0$ or $x(k+\ell)=0, \ell \in[i, N]$ what cannot occur for any nontrivial solution if $N \geq 2 n+1$ (i.e., the solution has at least two generalized zeros, [5]). The concepts of generalized zeros and disconjugacy might be directly generalized to difference equations of orders higher than two as follows.

\section{Definitions}

Definition 3.6. A solution of (2.5)-(2.6) has a generalized zero in $[k, k+N]$ for some $N \in \mathbb{N}_{1}$ if there exists an integer $i \in[k, k+N-1]$ such that $\delta(i, i+1) \leq 0$.

The generalized zero is weak if $\delta(i, j)<0$ for some integers $i \in[k, k+N-1], j(>i) \in$ $[k+1, k+N]$ and, furthermore, $\delta(\ell, \ell+1) \leq 0$ for $\ell \in[i, j-1]$.

The generalized zero is strong if $\delta(i, i+1)=\delta(i, i-1)=0 \Rightarrow x(i)=0$.

Definition 3.7. A solution of (2.5)-(2.6) is disconjugate in $[k, k+N]$ if there exist integers $i, j(>i), s(\geq j), r(>s) \in[k, k+N]$ such that $\delta(i, j)<0, \delta(s, r)<0$ with $\delta(\ell, \ell+1) \leq 0$ for $\ell \in[i, j-1]$ and $\delta(\ell, \ell+1) \leq 0$ for $\ell \in[s, r-1]$ (i.e., the solution possesses two weak generalized zeros in $[k, k+N])$.

Note that weak generalized zeros in some interval imply changes of sign of the solution and strong generalized zeros imply zeros of the solution. The property of disconjugacy implies and is implied by the presence of two weak generalized zeros within the same interval. The subsequent results rely on the properties of disconjugacy and generalized zeros.

Proposition 3.8. If a solution of (2.5)-(2.6) is disconjugate and nontrivial in $[k, \infty)$ for $k \geq n-1$, then it is GWO and GO.

Proof. Since the solution is nontrivial, then for all $[k+i, k+i+N-1], i \in[0, \infty)$, then there is $\ell, j \in[i-1, i+N-2]$ such that $x(j+1) \neq 0, \delta\left(\ell+1, \ell+\ell^{\prime}+1\right)<0$ with $x(j)=0$ or $\delta(j, \ell) \neq 0$. Since it is disconjugate and nontrivial, $\delta\left(j+1, j+j^{\prime}\right)<0$ so that it $\mathrm{SO}$ and WO in $[k, k+n-1]$ and the solution is nontrivial. The reasoning is identical on $[k+1, \infty)$ 
for intervals $[k+i, k+i+n+1]$ for all $i \in[0, \infty)$ and some $j \in[i+1, i+n-2]$ so that the solution is GSO and then GWO.

THeOREM 3.9. The following properties hold for any solution $\{x(k)\}_{n}^{\infty}$ of (2.5)-(2.6) subject to initial conditions $x(i)=x_{i}, i \in[0, n-1]$ :

(i) it is WO in $[k, k+N]$, for all $k \in \mathbb{N}_{n}$ if and only if it is disconjugate in $[k, k+N]$, for all $k \in \mathbb{N}_{n}$;

(ii) it is GWO in $[k, k+N]$, for all $k \in \mathbb{N}_{n}$ if and only if there exists an integer $N(k) \in$ $[k+2, \bar{N}]$ with $\bar{N}:=\operatorname{Sup}_{k \geq n}(N(k))<\infty$ such that it is disconjugate in $[k, k+N(k)]$, for all $k \in \mathbb{N}_{n}$;

(iii) it is GSSO if and only if each interval $[k, k+1]$ has a weak generalized zero, for all $k \in \mathbb{N}_{n}$

(iv) it is GSSO if and only if it is disconjugate in each interval $[k, k+2]$, for all $k \in \mathbb{N}_{n}$;

(v) it is GSO if and only if it is disconjugate in each interval $[k, k+n]$, for all $k \in \mathbb{N}_{n}$.

Proof. (i) "If part" If the solution is disconjugate in $[k, k+N]$, then there exist integers $\mathrm{i}, j(>i), s(\geq j), r(>s) \in[k, k+N]$ implying $\delta(i, j)<0, \delta(s, r)<0$ with no sign changes in-between samples $(i, j)$ and in-between samples $(s, r)$ (Definition 3.7) implying that is WO in $\in[k, k+N]$ (Definition 3.1). The converse reasoning is similar to prove the "Only if part."

(ii) "If part" Assume that for each $k_{\sigma} \geq n$ there exists a finite $N\left(k_{\sigma}\right)$ and integers $i, j(>i), s(\geq j), r(>s) \in\left[k_{\sigma}, k_{\sigma}+N\left(k_{\sigma}\right)\right]$ such that $\delta(i, j)<0, \delta(s, r)<0$ with no sign changes in-between samples $(i, j)$ and in-between samples $(s, r)$. Thus, the solution is disconjugate in $\left[k_{\sigma}, k_{\sigma}+N\left(k_{\sigma}\right)\right]$. By construction, note that $\mathbb{N}_{n}=\bigcup_{\sigma=1}^{\infty}\left[k_{\sigma}, k_{\sigma}+N\left(k_{\sigma}\right)\right]=$ $\bigcup_{k_{i} \in \mathbb{N}_{n}}^{\infty}\left[k_{i}, k_{i}+N\left(k_{i}\right)\right]$, the first union of intervals being countable, the second one being uncountable, provided that $k_{\sigma+1}=k_{\sigma}+N\left(k_{\sigma}\right)+1$ with $k_{\sigma} \in \mathbb{N}_{n}$ for each $\sigma \in \mathbb{N}_{1}$. Since the solution has two generalized zeros within each finite subinterval of such unions because it is disconjugate, it follows that it is GWO and sufficiency has been proved. ("Only if part") Proceed by contradiction by assuming that there is an interval $[k, \infty)$ without two weak generalized zeros. Then, the solution cannot be GWO.

(iii) “If part” $\delta(k, k+1)<0$, for all $k \in \mathbb{N}_{n} \Rightarrow \delta(k+i, k+i+1)<0$ for $i=0,1 ;[k, k+2]$ so that the solution has two weak generalized zeros and then two sign changes within each interval $[k, k+2]$. ("Only if part.") Proceed by contradiction. Assume that there is an interval $[k, k+1]$ for some $k \in \mathbb{N}_{n}$ such that $\delta(k, k+1) \geq 0$. Then the solution has either a strong zero at $k$ or $k+1$ or two nonzero values of the same sign.

(iv) If the solution is disconjugate in $[k, k+2]$, it has two sign changes and then two generalized weak zeros from Definition 3.7 within such an interval. Since the property holds for all $k \in \mathbb{N}_{n}$, the solution is GSSO. The necessity part is proved by contradiction arguments in a similar way as the "If part" of property (iii) for either $\delta(k, k+1) \geq 0$ or $\delta(k+1, k+2) \geq 0$.

(v) The proof is similar to that of (iv) by using intervals $[k, k+n]$ for all $k \in \mathbb{N}_{n}$ and Definition 3.6.

Proposition 3.10. A solution of (2.5)-(2.6) with $x(i)=x_{i}, i \in[0, n-1]$, which is not disconjugate in $[k, k+N]$ for some $k \geq n$, has at most one sign change in $[k, k+N]$ for $k \geq n$. 
It has no generalized zeros in $[k, k+N]$ for some $k \geq n$ if and only if it is sign-constant in $[k, k+N]$ for $k \geq n$.

The proof of Proposition 3.10 is omitted. Note that the results about oscillations, disconjugacy, and generalized zeros might be also discussed from the associate dynamic system (2.7) since

$$
\delta(i, j)=\delta(j, i)=e_{1}^{T} z(j) z^{T}(i) e_{1}=e_{1}^{T} z(i) z^{T}(j) e_{1}=e_{\ell}^{T} z(j-\ell+1) z^{T}(i-r+1) e_{r},
$$

wherein $e_{\ell}$ and $e_{r}$ any $\ell, r \in[1, n]$. The following technical result concerning sign changes and disconjugacy, whose proof is omitted, is stated.

Lemma 3.11. The difference equation (2.5)-(2.6) possesses the following properties.

(i)

$$
\begin{aligned}
S(k-i+2, k+1) & :=\delta(k-i+2, k+1)+\sum_{j=1}^{n} \alpha_{j}(k) \delta(k-j+1, k-i+2)=0, \\
S^{0}(k-i+2, k+1) & :=S(k-i+2, k+1)-\alpha_{\ell}(k) \delta(k-\ell+1, k-i+2) \\
& =0 \text { if } \quad \alpha_{\ell}(k)=0, \\
& \leq 0 \text { if } \quad \alpha_{\ell}(k)>0, \\
& \geq 0 \text { if } \quad \alpha_{\ell}(k)<0,
\end{aligned}
$$

for any $k \in \mathbb{N}_{n-1}$ and any $i, \ell \in[1, n]$.

(ii) Assume that $\alpha_{j}(k) \leq 0, j \in[1, n]$, with (at least) one $\alpha_{\ell}(k)<0$, some $\ell \in[1, n]$, for all $k \in \mathbb{N}_{n-1}$.

Then the difference equation (2.5)-(2.6), and so the dynamic system (2.7), is positive, $[13,14]$, so that $z(k)>0$, for all $k \geq n$ (i.e., no component of $z(k)$ is negative and $z(k) \neq$ 0 , equivalently, $z(k) \in \mathbb{R}_{+}^{n}$ - the first closed orthant in $\mathbb{R}^{n}$ - and at least one component is positive) for any $z(n-1)>0$. There is no nontrivial globally disconjugate solution, no WO solution, and no GO solution of (2.5)-(2.6) for any initial conditions $z(n-1) \in \mathbb{R}_{+}^{n}$ and also for any initial conditions $z(n-1) \in \mathbb{R}_{-}^{n}:=\left\{z \in \mathbb{R}^{n}:(-z) \in \mathbb{R}_{+}^{n}\right\}$.

(iii) Assume that any of the subsequent conditions hold

(1) $\alpha_{j}(k)>0$, for all $j \in[1, n]$ if $x(k+1-j) \neq 0$ and arbitrary otherwise;

(2) $\alpha_{j}(k) \geq 0$, for all $j \in[1, n]$ with at least one $\alpha_{\ell(k)}(k)>0$ some $\ell=\ell(k) \in$ $[1, n]$, for all $k \in \mathbb{N}_{n-1}$ such that $x(k+1-\ell(k)) \neq 0$.

Then, any nontrivial solution $\{x(k)\}_{0}^{\infty}$ of (2.5)-(2.6) on $[n, \infty)$ subject to initial conditions $x(i+1)=x_{i}, i \in[0, n-1]$, has at least one sign change within each interval $[k+\ell+1, k+$ $N]$, for all $k \in \mathbb{N}_{n-1}, N \geq n+\ell+1$, for all $\ell \in[0, \infty)$. Also, any of such nontrivial solutions has at least three sign changes within each interval $[k+\ell+1, k+N]$, for all $k \in \mathbb{N}_{n-1}, N \geq$ $2 n+\ell+1$, for all $\ell \in[0, \infty)$ so that it is disconjugate.

The following result guarantees that the difference equation (2.5)-(2.6) is WO within intervals of larger measures than the equation order. 
Theorem 3.12. Assume that there exist natural numbers $(k+N(k))$ and $\left(k+N(k)+N^{\prime}(k)\right)$ with $\operatorname{Min}\left(N(k)+N^{\prime}(k)\right) \geq n$, some $k \in \mathbb{N}_{n}$ such that

$$
\begin{aligned}
& \left\{i, j \in[1, n] \times[1, n]: \alpha_{i}(k+N(k)) \tilde{\mathbf{A}}_{i j}(k, k+N(k)-1)>0\right\} \\
& \quad \cap\left\{i, j \in[1, n] \times[1, n]: \alpha_{i}(k+N(k)) \tilde{\mathbf{A}}_{i j}\left(k+N(k), k+N(k)+N^{\prime}(k)-1\right)>0\right\} \neq \varnothing .
\end{aligned}
$$

Then, the following properties hold.

(i) Any nontrivial solution of the linear difference equation (2.5)-(2.6) is WO in $[k+$ $N(k), k+N(k)+N^{\prime}(k)$ ] if the following conditions hold:

(C1) $\widetilde{\mathbf{A}}_{1 \ell}(k, k+N(k))=-\sum_{i=1}^{n} \alpha_{i}(k+N(k)) \tilde{\mathbf{A}}_{i \ell}(k, k+N(k)-1)<0$, some $\ell \in$; and $\tilde{\mathbf{A}}_{1 \ell}\left(k+N(k), k+N(k)+N^{\prime}(k)\right)=-\sum_{i=1}^{n} \alpha_{i}\left(k+N(k)+N^{\prime}(k)\right) \tilde{\mathbf{A}}_{i \ell^{\prime}}(k+$ $\left.N(k), k+N(k)+N^{\prime}(k)-1\right)<0$, some $\ell^{\prime} \in[1, n]$

(C2) $\widetilde{\mathbf{A}}_{1 j}(k, k+N(k))=-\sum_{i=1}^{n} \alpha_{i}(k+N(k)) \tilde{\mathbf{A}}_{i \ell}(k, k+N(k)-1) \leq 0$, for all $j(\neq$ $\ell) \in[1, n]$ such that $x(k+N(k)+1-j) \neq 0$; and $\tilde{\mathbf{A}}_{1 \ell}(k+N(k), k+N(k)+$ $\left.N^{\prime}(k)\right)=-\sum_{i=1}^{n} \alpha_{i}\left(k+N(k)+N^{\prime}(k)\right) \tilde{\mathbf{A}}_{i \ell^{\prime}}\left(k+N(k), k+N(k)+N^{\prime}(k)-1\right) \leq$ 0 , for all $j(\neq \ell) \in[1, n]$ such that $x\left(k+N(k)+N^{\prime}(k)+1-j\right) \neq 0$.

(ii) Property (i) holds if (C2) is extended to all $\tilde{\mathbf{A}}_{1 j}(k, k+N(k))$ for all $j(\neq \ell) \in[1, n]$, $\tilde{\mathbf{A}}_{1 j}\left(k+N(k), k+N(k)+N^{\prime}(k)\right)$ for all $j\left(\neq \ell^{\prime}\right) \in[1, n]$.

(iii) Property (i) holds if there exists nonzero $\widetilde{\mathbf{A}}_{1 \ell}(k, k+N(k)-1)$ and $\widetilde{\mathbf{A}}_{1 \ell^{\prime}}(k+N(k), k+$ $\left.N(k)+N^{\prime}(k)-1\right)$, some $\ell, \ell^{\prime} \in[1, n]$ and the two following constraints hold:

$$
\alpha_{i}(k+N(k))>-\frac{\sum(i \neq j) \in N Z_{\ell}(k, k+N(k)-1)}{\tilde{\mathbf{A}}_{i \ell}(k, k+N(k)-1)}
$$

for some $\ell \in[1, n]$ and some $i \in N Z_{\ell}(k, k+N(k)-1)$ where

$$
\begin{aligned}
{[1, n] } & \supseteq N Z_{\ell}(k, k+N(k)-1): \\
& =\left\{i \in[1, n]: \alpha_{i}(k+N(k)) \tilde{A}_{1 \ell}(k, k+N(k)-1) \neq 0\right\} ;
\end{aligned}
$$

$\alpha_{i}\left(k+N(k)+N^{\prime}(k)\right)$

$$
>-\frac{\sum_{(i \neq j) \in N Z_{\ell}\left(k+N(k), k+N(k)+N^{\prime}(k)-1\right)} \alpha_{j}\left(k+N(k)+N^{\prime}(k)\right) \widetilde{\mathbf{A}}_{j \ell}\left(k+N(k), k+N(k)+N^{\prime}(k)-1\right)}{\widetilde{\mathbf{A}}_{i \ell}\left(k+N(k), k+N(k)+N^{\prime}(k)-1\right)}
$$

for some $\ell \in[1, n]$ and some $i \in N Z_{\ell}\left(k+N(k), k+N(k)+N^{\prime}(k)-1\right)$.

(iv) Assume that $\operatorname{Min}\left(N(k), N^{\prime}(k)\right) \geq n$. Then, if property (i) holds for each one of the intervals $[k, k+N(k)],\left[k+N(k)+2, k+N(k)+N^{\prime}(k)+3\right]$, for all $k \in \mathbb{N}_{n}$, then any solution of (2.5)-(2.6) is GWO.

Proof. (i) It follows from (2.2), Lemma 3.11(ii), and the recursive identities (2.13) since the set which is defined in (3.3) is nonempty, $\widetilde{\mathrm{A}}_{1 \ell}(k, k+N(k))<0$ some $\ell \in[1, n]$ and $\tilde{\mathbf{A}}_{1 \ell}\left(k+N(k), k+N(k)+N^{\prime}(k)-1\right)<0$ some $\ell \in[1, n]$. Thus, (C1)-(C2) imply that there 
are at least two generalized weak zeros in $\left[k, k+N(k)+N^{\prime}(k)\right]$, one being located in $[k, k+N(k)]$ and another one in $\left[k+N(k)+1, k+N(k)+N^{\prime}(k)\right]$ so that the solution id WO in $\left[k, k+N(k)+N^{\prime}(k)\right]$. The proof of property (ii) is similar since the $\tilde{\mathbf{A}}_{1 \ell}(\cdot)$ corresponding to parameters associated with $x(k+N(k)+1-j)=0$ do not contribute to the solution. Property (iii) follows immediately from property (i). Property (iv) is direct since property (i) holds for all $k \in \mathbb{N}_{n}$.

\section{Oscillations under unmodeled dynamics}

4.1. Conditions of existence of oscillatory solutions. The tests of results about oscillations of the solutions of the above section require some "a priori" knowledge (consisting at least of their signs and the whole equation order) on all the time-varying parameters $\alpha_{i}(k)$ in order to elucidate if the solution oscillates or not. Now, sufficient-type conditions of oscillation of the solution of the current difference equation of $n$ th-order are related to those of its nominal part of order $n_{0}$ with constraints on the unmodeled one. The study is performed via the companion dynamic system when necessary. Assume that $e_{0 i}$ and $e_{i}$ are the $i$ th unity vectors of $\mathbb{R}^{n_{0}}$ and $\mathbb{R}^{n}$. From (2.5) and (2.10)-(2.11), one gets directly by relating the first components of the associated dynamical system with the solution sequence of (2.5)-(2.6):

$$
\begin{aligned}
\delta(k, k+N(k)+1) & =e_{1}^{T} \tilde{z}(k+N(k)+1) \tilde{z}^{T}(k) e_{1}=e_{1}^{T} \tilde{\mathbf{A}}(k, k+N(k)) \tilde{z}(k) \tilde{z}^{T}(k) e_{1} \\
& =e_{01}^{T}\left(\mathbf{A}(k, k+N(k)) z(k) z^{T}(k)+\boldsymbol{\eta}(k, k+N(k)) z^{T}(k)\right) e_{01} \\
& =e_{01}^{T} \mathbf{A}(k, k+N(k)) z(k) z^{T}(k) e_{01}+e_{01}^{T} \boldsymbol{\eta}(k, k+N(k)) z^{T}(k) e_{01} \\
& \leq e_{01}^{T} \mathbf{A}(k, k+N(k)) z(k) z^{T}(k) e_{01}+\bar{\eta}(k, k+N(k))|x(k)|,
\end{aligned}
$$

where $\bar{\eta}(k, k+N(k)) \geq\left|\eta_{1}(k, k+N(k))\right|=\left|e_{1}^{T} \boldsymbol{\eta}(k, k+N(k))\right|$ is an available absolute upper-bound of the first component $\boldsymbol{\eta}_{1}(k, k+N(k))$ of $\boldsymbol{\eta}(k, k+N(k))$. The problem of oscillations under unmodeled dynamics is formalized in the subsequent result.

Theorem 4.1. The following two properties hold:

(i) assume that $x(k) \neq 0$ and $x(k+N(k)+1-i) \neq 0$ for some $k \in \mathbb{N}_{n}$ and for some $i \in$ $[1, n]$, which always exists for any nontrivial solution $\{x(k)\}_{0}^{\infty}$ of (2.5)-(2.6) under nonidentically zero initial conditions $x(i)=x_{i} \in \mathbb{R}, i \in[0, n-1]$. Then, $\delta(k, k+$ $N(k)-1)<0$ if and only if

$$
\alpha_{i}(k, k+N(k))>\bar{\eta}(k+N(k))-\sum_{(i \neq) j=1}^{n} \frac{\alpha_{j}(k+N(k)+1) x(k+N(k)+1-j)}{x(k) x(k+N(k)+1-i)} ;
$$

(ii) a sufficient condition for (4.2) to hold is

$$
\begin{aligned}
\alpha_{i}(k, k+N(k))>\left(\bar{n}-n_{0}\right) \bar{\alpha}(j) \operatorname{Max}_{n_{0}<i \leq \bar{n}}(|x(k+N(k))-i|) & \\
& -\sum_{(i \neq) j=1}^{n} \frac{\alpha_{j}(k+N(k)+1) x(k+N(k)+1-j)}{x(k) x(k+N(k)+1-i)} \operatorname{Sign}(x(k))
\end{aligned}
$$


for some available known integer constant $\bar{n} \geq n$ and $\bar{\alpha}(j)$ defined as

$$
\bar{\alpha}(j)=\sum_{i=n_{0}+1}^{\bar{n}}\left|\alpha_{n_{0}+i}^{0}(j)\right| ; \quad \tilde{\alpha}(j)=\sum_{i=1}^{n_{0}}\left(\left|\alpha_{i}^{0}(j)\right|+\sum_{i=1}^{n_{0}} \bar{\alpha}_{i}(j)\right) .
$$

Proof. (i) Follows directly from the expression

$$
\delta(k, k+N(k)+1) \leq-\sum_{j=1}^{n} \alpha_{j}(k+N(k)) \delta(k, k+N(k)+1-j)+|\bar{\eta}(k+N(k)) x(k)|<0
$$

provided that (4.3) holds. Property (ii) follows directly from property (i).

Note that (4.3) might be achieved with the knowledge of any $\bar{n} \geq n$. Computations of lower-bounds of the values of $\bar{\alpha}(j)$ lead to weaker conditions of fulfilment of Theorem 4.1(i) which will yield later weaker oscillation conditions for the difference equation (2.5)-(2.6). The subsequent result, whose proof is omitted, follows from Theorem 4.1 and Definition 3.1.

Theorem 4.2. Consider sequences of integer numbers $\mathbf{S}_{\alpha}:=\left\{k_{i}\right\}_{n}^{\infty}, \mathbf{S}_{\beta \mathrm{N}}:=\left\{N\left(k_{i}\right)\right\}_{n}^{n_{s}}$ fulfilling the constraint $\infty>n_{S} \geq N\left(k_{i}\right):=k_{i+1}-k_{i} \geq n$ for some finite integer $n_{S} \geq n$. Then,

(i) assume that $e_{01} \in \operatorname{Ker}\left(I_{n_{0}}+\lambda\left(k_{i}\right) \mathbf{A}^{T}\left(k_{i}, k_{i+1}\right)\right)$ and $\lambda\left(k_{i}\right)>\left(\left|x\left(k_{i}\right)\right| \overline{\boldsymbol{\eta}}\left(k_{i}, k_{i+1}\right)\right) /$ $\left(\left\|\mathbf{A}^{T}\left(k_{i}, k_{i+1}\right) z\left(k_{i}\right)\right\|_{2}^{2}\right)$ for all $k_{i} \in \mathbf{S}_{\boldsymbol{\alpha}}$ with $\left(k_{i+1}-k_{i}\right) \in \mathbf{S}_{\beta \mathrm{N}}$, for all $i \in \mathbb{N}_{n}$. Then, $\delta\left(k_{i}, k_{i+1}\right)<0$ and any nontrivial solution of (2.5)-(2.6) is GWO;

(ii) if

$$
\alpha_{\ell}\left(k_{i+1}\right)>\bar{\eta}\left(k_{i+1}\right)-\sum_{(\ell \neq) j=1}^{n} \frac{\alpha_{j}\left(k_{i+1}\right) x\left(k_{i+1}+1-j\right) \bar{\eta}\left(k_{i}, k_{i+1}\right)}{x\left(k_{i}\right) x\left(k_{i+1}+1-\ell\right)} \operatorname{Sign}\left(x\left(k_{i}\right)\right)
$$

then, $\delta\left(k_{i}, k_{i+1}+1\right)<0$ and any nontrivial solution of (2.5)-(2.6) is GWO.

4.2. Oscillatory stable solutions. In this subsection combined results are discussed concerning the existence of oscillatory solutions being globally (Lyapunov) stable (i.e., uniformly bounded), globally asymptotically stable (i.e., stable and converging asymptotically to zero) and globally exponentially stable (i.e., globally asymptotically stable with exponential rate of convergence). Those stability properties imply those parallel ones of the associate dynamic system (2.7). Note from (2.10) that

$$
z(k+N(k)+1)=\left(\prod_{i=k}^{k+N(k)}[A(i)]\right) z(k)+\sum_{j=k}^{k+N(k)} \prod_{i=j+1}^{k+N(k)}[A(i)] \eta(k)
$$

The following assumptions are then used to discuss stability results.

Assumption 4.3. The nominal equation (2.5) (i.e., $\eta(k) \equiv 0$ ) is globally exponentially stable. 
Assumption 4.3 holds if and only if $\{A(k)\}_{0}^{\infty}$ is a convergent sequence of matrices; that is, there exist (norm-dependent) real constants $K \geq 1$ and $\rho \in(0,1)$ such that $\|\mathbf{A}(i, j)\| \leq$ $K \rho^{j-i}$, for all $i \in \mathbb{N}_{n-1}, k \in \mathbb{N}_{i+1}$.

Assumption 4.4. The sequence $\eta(k)=\theta_{\mathrm{um}}^{T}(k) \varphi(k) \rightarrow 0$ as $k \rightarrow \infty$.

Two typical situations in which Assumption 4.4 holds are $\theta_{\text {um }}(k) \rightarrow 0$ as $k \rightarrow \infty$ with $\left\{\theta_{\text {um }}(k)\right\}_{0}^{\infty}$ uniformly bounded and $\left\{\theta_{\text {um }}(k)\right\}_{0}^{\infty}$ being uniformly bounded with $\varphi(k) \rightarrow 0$ as $k \rightarrow \infty$. The following result holds.

Proposition 4.5. If Assumption 4.3 holds, then the difference equation (2.5)-(2.6) is globally Lyapunov stable so that its associate dynamic system (2.7)-(2.8) is globally Lyapunov stable for sufficiently small $\left\|\theta_{\mathrm{um}}(k)\right\|$, for all $k \in \mathbb{N}_{k_{0}}$, and for some finite $k_{0} \in \mathbb{N}_{n-1}$.

Proof. From (2.10) and Assumption 4.3, one gets

$$
\|z(k+N(k)+1)\| \leq K\left[\rho^{N(k)+1}\|z(k)\|+\frac{\gamma(k, N(k))}{1-\rho} \operatorname{Sup}_{1 \leq j \leq N(k)+n}(\|z(k+N(k)-j)\|)\right] .
$$

Since $\sum_{k=0}^{\infty} \rho^{k}=1 / 1-\rho$ is a convergent series for $\rho \in(0,1) \cap \mathbb{R}$, then

$$
K \sum_{j=k}^{k+N(k)} \rho^{k+N(k)-j}=K \sum_{j=0}^{N(k)} \rho^{N(k)-j}=\frac{1-\rho^{N(k)+1}}{1-\rho},
$$

where $\gamma(k, N(k))>0$ is a norm-dependent real constant which depends on the $\operatorname{Sup}_{k \leq j \leq k+N(k)}\left(\left\|\theta_{\text {um }}(j)\right\|\right)$. If $\mu(k, N(k)):=K\left[\rho^{N(k)+1}+(\gamma(k, N(k)) / 1-\rho)\right] \leq 1$, what always occurs for all $N(k) \geq N_{*}$ and any $K \geq 1$, and if $\gamma_{0}:=\operatorname{Sup}_{j \geq k}(\gamma(k, N(k)))$ is sufficiently small, what holds if $\left\|\theta_{\mathrm{um}}(j)\right\|$ is sufficiently small for $j \in[k, k+N(k)]$, then

$$
\|z(k+N(k)+)\|_{2} \leq \mu(k, N(k)) \operatorname{Sup}_{k-n \leq j \leq k+N(k)}\left(\|z(j)\|_{2}\right) \leq \operatorname{Sup}_{k-n \leq j \leq k+N(k)}\left(\|z(j)\|_{2}\right)
$$

what implies $\operatorname{Sup}_{k-n \leq j \leq k+N(k)+1}\left(\|z(j)\|_{2}\right) \leq \operatorname{Sup}_{k-n \leq j \leq k+N(k)}\left(\|z(j)\|_{2}\right)$ for all integer $k \geq$ $k_{0}$, some finite integer $k_{0} \geq n-1$ and all integer $N(k) \geq N_{*}$. As a result, the dynamic system (2.7)-(2.8) is globally Lyapunov stable, and the solution sequence $\{x(k)\}_{n-1}^{\infty}$ to the difference equation (2.5) is uniformly bounded, for any bounded initial conditions $\widetilde{z}(n-$ 1) $\in \mathbb{R}^{n}$ provided that $\left\|\theta_{\mathrm{um}}(j)\right\|$ is sufficiently small for all $k \geq k_{0}$ and Assumption 4.3 holds.

The proof of the subsequent result is omitted

Proposition 4.6. The following properties hold provided that Assumptions 4.3-4.4 hold:

(i) the solution of the difference equation (2.5)-(2.6) is bounded and tends asymptotically to zero as $k \rightarrow \infty$. Its associated dynamic system is globally asymptotically Lyapunov stable; 
(ii) if $\eta(k) \rightarrow 0$ exponentially as $k \rightarrow \infty$, then $x(k) \rightarrow 0$ as $k \rightarrow \infty$ and its associate $d y$ namic system is globally exponentially stable.

The main result concerning the existence of stable oscillations is stated.

Theorem 4.7. The subsequent properties hold.

(i) The time-varying difference equation (2.5)-(2.6) is globally asymptotically stable if Assumption 4.3 holds and, furthermore, for some positive definite monotonically nondecreasing $n_{0}$-matrix sequence $\{P(k)\}_{n-1}^{\infty}=\left\{P^{(i j)}(k)\right\}_{n-1}^{\infty}$, with $P(k)$ being of columns $P^{(i)}(k)$, and $\bar{\eta}(k)$ satisfing the following joint constraints:

$$
\begin{aligned}
& \mathbf{A}^{T}(k, k+N(k)) P(k+N(k)+1) \mathbf{A}(k, k+N(k))-P(k)<0, \\
& \bar{\eta}(k, k+N(k)) \leq-2 z^{T}(k) \mathbf{A}^{T}(k, k+N(k)) P(k+N(k)+1)
\end{aligned}
$$

for all integer $k \geq k_{0}$, some nonnegative finite integer $k_{0}$, and some sequence of positive finite integers $\{N(k)\}_{0}^{\infty}$ dependent on $k$;

(ii) $\delta(k, k+N(k)-1)<0$ for any combination of parameters $\alpha_{i}(k+N(k)-j), j \in$ $[0, n-1], i \in[1, n]$ satisfying the set of constraints

$$
\begin{array}{r}
-\widetilde{\mathbf{A}}_{11}(k, k+N(k))=\sum_{i=1}^{n} \alpha_{i}(k+N(k)) \widetilde{A}_{i 1}(k, k+N(k)-1)=\frac{1}{\lambda(k)}, \\
-\widetilde{\mathbf{A}}_{1, i+1}(k, k+N(k)-i)=\sum_{j=1}^{n} \alpha_{j}(k+N(k)-i) \tilde{A}_{j, i+1}(k, k+N(k)-i-1)=0, \\
i \in[1, n-1],
\end{array}
$$

provided that

$$
\begin{aligned}
\bar{\eta}(k, k+N(k)) & <\left(\frac{\left\|\mathbf{A}^{T}(k, k+N(k)) z(k)\right\|_{2}^{2}}{\|z(k)\|_{2}^{2}}\right)\left(\left\|\mathbf{A}^{T}(k, k+N(k)) z(k)\right\|_{2}^{2}\right) \\
& <\frac{\left\|\mathbf{A}^{T}(k, k+N(k)) z(k)\right\|_{2}^{2}}{|x(k)|}
\end{aligned}
$$

since $\|z(k)\| \geq|x(k)|$. Also, since $\bar{\eta}(k, k+N(k)) \leq \gamma_{\eta} \operatorname{Sup}_{k-n_{a} \leq j \leq k+N(k)-1-n_{a}}(\|z(j)\|)$ with $n_{a}:=\operatorname{Min} \operatorname{Int}\left(\ell \geq n-n_{0}\right)$ and $\gamma_{\eta}\left\{\begin{array}{l}>0 \text { if } n>n_{0} \\ =0\end{array}\right.$ if $n=n_{0}$, a sufficient condition for (4.13) and (4.14) to hold is

$$
\begin{aligned}
\operatorname{Sup}_{k-n_{a} \leq j \leq k+N(k)-1-n_{a}}(\|z(j)\|) & \leq \frac{1}{\gamma_{\eta}}\left(\frac{\left\|\mathbf{A}^{T}(k, k+N(k)) z(k)\right\|_{2}^{2}}{|x(k)|}\right), \\
\operatorname{Sup}_{k-n_{a} \leq j \leq k+N(k)-1-n_{a}}(\|z(j)\|) & \leq \frac{1}{\gamma_{\eta}}\left(\frac{\left\|\mathbf{A}^{T}(k, k+N(k)) z(k)\right\|_{2}^{2}}{\|z(k)\|_{2}^{2}}\right) ;
\end{aligned}
$$


(iii) $\delta(k, k+N(k)-1)<0$ for any $\lambda(k)>|x(k)| \bar{\eta}(k, k+N(k)) /\left\|\mathbf{A}^{T}(k, k+N(k)) z(k)\right\|_{2}^{2}$ provided that the set $\alpha_{i}(k, k+N(k)), i \in[1, n]$, satisfies uniquely the constraints

$$
\begin{aligned}
\boldsymbol{\alpha}(k+N(k)): & =\left(\alpha_{1}(k+N(k)), \alpha_{2}(k+N(k)), \ldots, \alpha_{n}(k+N(k))\right) \\
& =-\lambda^{-1}(k+N(k)) \tilde{\mathbf{A}}^{-T}(k, k+N(k)) e_{1}
\end{aligned}
$$

provided that $\tilde{\mathbf{A}}(k, k+N(k))$ is nonsingular.

If the above constraints for $\lambda(k)$ and $\boldsymbol{\alpha}(k)$ hold for any $k \geq n-1$ and some sequence $\{N(k) \geq n\}_{n-1}^{\infty}$, then the system (2.7) is GWO.

If, in addition, there is a nondecreasing sequence of symmetric positive definite $n_{0}$-matrices $\{P(k)\}_{n-1}^{\infty}$ (trivially including the particular case of constant positive definite $P(k)=P=P^{T}$ ) such that (4.11a) holds, then any solution sequence $\{x(k)\}_{n}^{\infty}$ of the difference equation (2.5)-(2.6) is bounded and converges asymptotically to zero for any bounded initial conditions $\{x(k)\}_{0}^{n-1}$.

If $\lambda(k)>|x(k)| \bar{\eta}(k, k+N(k)) /\left\|\mathbf{A}^{T}(k, k+N(k)) z(k)\right\|_{2}^{2}$ and, furthermore, there is a nondecreasing sequence of symmetric positive definite n-matrices $\{\tilde{P}(k)\}_{n-1}^{\infty}$ (trivially including the particular case of constant positive definite $\widetilde{P}(k)=\widetilde{P}=\widetilde{P}^{T}$ ) such that the subsequent Lyapunov matrix inequality

$$
\widetilde{\mathbf{A}}^{T}(k, k+N(k)) \widetilde{P}(k+N(k)+1) \tilde{\mathbf{A}}(k, k+N(k))-\widetilde{P}(k)<0
$$

holds for all integer $k \geq k_{0}$, some nonnegative finite integer $k_{0}$, and some sequence of positive finite integers $\{N(k)\}_{0}^{\infty}$ dependent on $k$, then any solution sequence $\{x(k)\}_{n}^{\infty}$ of the difference equation (2.5)-(2.6) is bounded and converges asymptotically to zero for any bounded initial conditions $\{x(k)\}_{0}^{n-1}$.

Proof. (i) The tentative Lyapunov function candidate $V(k)=z^{T}(k) P(k) z(k)$ is a Lyapunov function for the dynamic system (2.7) if (4.10) hold guaranteeing that $\| z(k+$ $N(k))\left\|_{2}<\right\| z(k) \|_{2}$, for all $k \geq k_{0}$, with the sequence $\|z(k+N(k))\|_{2}$ being uniformly bounded and monotonically decreasing (and thus converging asymptotically to zero as $k \rightarrow \infty)$. Since the sequence $\{N(k)\}_{0}^{\infty}$ consists of uniformly bounded integer numbers, $z(j)$ cannot diverge within each interval $[k, k+N(k)]$, for all $k \geq k_{0}$ under bounded initial conditions. Property (i) has been proved.

(ii)-(iii) (Outline). The proof follows since the solution of (2.5)-(2.6) is oscillatory. Since (4.10) hold, the solution is globally asymptotically stable from property (i). The proof of property (ii) follows directly. The proof of property (iii) is similar by considering the associate dynamic system to (2.5)-(2.6) as perfectly modeled (i.e., of dimension $n$ described by (2.8)-(2.9)) since (4.17) ensures global Lyapunov asymptotic stability.

Note that (4.15)-(4.16) are feasible if the dynamic system (2.7) is globally Lyapunov stable. 


\section{Examples}

Example 5.1 ( $n$th time-varying difference equation under nominal parametrical uncertainties and unmodeled dynamics). Consider the $n$-order time-varying difference equation of nominal order $n_{0} \in[1, n]$ :

$$
x(k+1)=-\sum_{j=1}^{n} \alpha_{j}(k) x(k+1-j)=-\sum_{j=1}^{n_{0}} \alpha_{j}^{0}(k) x(k+1-j)+\eta(k)
$$

and $\eta(k)=\sum_{j=1}^{n_{0}}\left(\alpha_{j}^{0}(k)-\alpha_{j}(k)\right) x(k+1-j)-\sum_{j=n_{0}+1}^{n} \alpha_{j}(k) x(k+1-j)$ is a measurable unknown sequence describing unmodeled dynamics of order $\left(n-n_{0}\right)$ and parametrical errors. Note that for all integer $i \in[0, n-1]$ :

$$
\delta(k-i, k+1)=-\alpha_{i}^{0}(k) x^{2}(k-i)-\sum_{i+1 \neq i=1}^{n_{0}} \alpha_{j}^{0}(k) x(k+1-j) x(k-i)+\eta(k) x(k-i) .
$$

Note that for each integer $k \geq n_{0}$, there always exists (at least) an integer $i(k) \in[0, n-1]$ such that $x(k-i) \neq 0$ for any everywhere nontrivial solution associated with arbitrary non identically zero initial conditions $x(i) \in \mathbb{R}$. For such an $i(k), \delta(k-i(k), k+1)<0$ so that the solution possess a weak generalized zero within $[k-n, k+1]$ if

$$
\alpha_{i(k)+1}^{0}(k)>\frac{\eta(k)-\sum_{i(k) \neq j=1}^{n_{0}} \alpha_{j}^{0}(k) x(k+1-j)}{|x(k-i(k))|} .
$$

It is not guaranteed for any nontrivial solution and any $k$ that such an integer $i(k) \in$ $\left[0, n_{0}-1\right]$, that is, inside a nominal modeling interval. Note also that the above formula is still applicable if $i(k) \in\left[k-n_{0}-1, k+1\right]$. Absolute upper-bounds $\bar{\eta}(k) \geq|\eta(k)|$ might be computed from

$$
\begin{aligned}
|\eta(k)| \leq & \left|\sum_{j=1}^{n_{0}}\left(\alpha_{j}(k)-\alpha_{j}^{0}(k)\right) x(k+1-j)+\sum_{j=n_{0}+1}^{n} \alpha_{j}(k) x(k+1-j)\right| \quad \text { as, for instance, } \\
\bar{\eta}_{1}(k)= & \left(n_{0} \operatorname{Max}_{1 \leq j \leq n_{0}}\left(\left|\tilde{\alpha}_{j}(k)\right|\right)+\left(\bar{n}-n_{0}\right) \operatorname{Max}_{n_{0}+1 \leq j \leq \bar{n}}\left(\left|\alpha_{j}(k)\right|\right)\right) \operatorname{Sup}_{k \leq j \leq k+\bar{n}-1}(|x(j)|), \\
\bar{\eta}_{2}(k)= & \left(\sum_{j=1}^{n_{0}}\left|\tilde{\alpha}_{j}(k)\right|+\sum_{n_{0}+1}^{\bar{n}}\left(\bar{n}-n_{0}\right)\left|\alpha_{j}(k)\right|\right) \operatorname{Sup}_{k \leq j \leq k+\bar{n}-1}(|x(j)|), \\
\bar{\eta}_{3}(k)= & \left(n_{0} \operatorname{Max}_{1 \leq j \leq n_{0}}\left(\left|\tilde{\alpha}_{j}(k)\right|\right) \underset{k \leq j \leq k+1-n_{0}}{\operatorname{Sup}}(|x(j)|)\right. \\
& \left.+\left(\bar{n}-n_{0}\right) \operatorname{Max}_{n_{0}+1 \leq j \leq \bar{n}}\left(\left|\alpha_{j}(k)\right|\right)\right) \underset{k-n_{0} \leq j \leq k+1-\bar{n}}{\operatorname{Sup}_{M}}(|x(j)|),
\end{aligned}
$$




$$
\begin{aligned}
\bar{\eta}_{4}(k)= & \left(\sum_{j=1}^{n_{0}}\left|\tilde{\alpha}_{j}(k)\right|\right) \operatorname{Sup}_{k \leq j \leq k+1-n_{0}}(|x(j)|) \\
& +\left(\sum_{n_{0}+1}^{\bar{n}}\left(\bar{n}-n_{0}\right)\left|\alpha_{j}(k)\right|\right) \operatorname{Sup}_{k-n_{0} \leq j \leq k+1-\bar{n}}(|x(j)|)
\end{aligned}
$$

provided that the remaining nominal parameters are arbitrary where $\tilde{\alpha}_{j}(k)=\alpha_{j}(k)-$ $\alpha_{j}^{0}(k), j \in\left[1, n_{0}\right]$, are parametrical errors of the modeled part and $\bar{n} \geq n$ is a known upper-bound of the equation order. Thus, at least one sign change in the solution (and then a generalized weak zero) is guaranteed within the interval $[k-n+1, k+1]$ if any of the conditions below holds:

$$
\alpha_{i(k)+1}^{0}(k)>\frac{\bar{\eta}_{\ell}(k)-\sum_{i(k) \neq j=1}^{n_{0}} \alpha_{j}^{0}(k) x(k+1-j)}{|x(k-i(k))|}, \quad \text { some } \ell \in[1,4]
$$

for at least one $i(k) \in[0, n-1]$ and the remaining parameters being arbitrary. If the above condition holds for some $i(k) \in[0, n-1]$ for all integer $k \geq n$, then the solution is GO and then globally disconjugate. The solution is also GO under the weaker condition that the integer $i\left(k_{i}\right) \in[0, n-1]$ such that $x\left(k-i\left(k_{i}\right)\right) \neq 0$ is guaranteed only for a sequence $\left\{k_{i}\right\}_{1}^{\infty}$ with $k_{1} \in\left[\bar{n}, \bar{n}+\bar{k}_{1}\right]$ and $1 \leq k_{i+1}-k_{i} \leq \bar{k}<\infty$. On the other hand, if

$$
\alpha_{i(k)+1}^{0}(k)>\frac{\bar{\eta}_{\ell}(k)-\sum_{i(k) \neq j=1}^{n_{0}} \alpha_{j}^{0}(k) x(k+1-j)}{|x(k-i(k))|}, \quad \text { some } \ell \in[1,4],
$$

then the stronger condition that the difference equation is GSSO holds:

$$
\alpha_{i+1}^{0}(k)>\frac{\chi_{i+1}(k)+\bar{\eta}_{\ell}(k)-\sum_{i \neq j=1}^{n_{0}} \alpha_{j}^{0}(k) x(k+1-j)}{|x(k-i)|}, \quad \forall i \in[0, \bar{n}-1] \text { and some } \ell \in[1,4]
$$

for all $k \in \mathbb{N}_{n}$ for any bounded sequences $\left\{\chi_{i+1}(k) \geq 0\right\}_{n}^{\infty}$ such that $\prod_{i=1}^{\bar{n}}(x(k+i)+$ $g(k+i-1)) \neq 0$ for all sequences $\{g(k) \in[-\bar{\eta}(k), \bar{\eta}(k)]\}_{n}^{\infty}$, since this implies that $x(k+$ $1) \neq 0$ for each nonzero $x(k)$ with $\delta(k, k+1)<0$, for all $k \in \mathbb{N}_{n}$. Any nontrivial solution of the nominal difference equation is GO if for some integer $i(k) \in[0, n-1]$ (which always exists) and all $k \in \mathbb{N}_{n}$ :

$$
\alpha_{i(k)+1}^{0}(k)>-\frac{\sum_{i(k) \neq j=1}^{n_{0}} \alpha_{j}^{0}(k) x(k+1-j)}{|x(k-i(k))|} .
$$

Note that if $\alpha_{j}(k) \leq 0, j \in[1, n]$, for all $k \in \mathbb{N}_{n}$, with at least one $\alpha_{i(k)}(k)<0$, for all $k \in \mathbb{N}_{n}$, the associated dynamic system is positive and no solution oscillates for any set of initial conditions $0 \leq \tilde{z}(0) \in \mathbb{R}^{n}$ or $0 \geq \tilde{z}(0) \in \mathbb{R}^{n}$.

Example 5.2 (reparameterization of Example 5.1 with unknown $n$ ). Example 5.1 might be described equivalently as follows through calculating by a difference equation the 
amount $x(k)$ :

$$
\begin{aligned}
x(k+1)= & -\sum_{j=0}^{n-1} \alpha_{j+1}(k) x(k-j)=\sum_{j=1}^{n-1}\left(\alpha_{1}(k) \alpha_{j}(k-1)-\alpha_{j+1}(k)\right) x(k-j) \\
& +\alpha_{1}(k) \alpha_{n}(k-1) x(k-n) .
\end{aligned}
$$

Then,

$$
\delta(k-1, k+1)=-\sum_{j=1}^{n} \alpha(k-1, k) \delta(k-j, k-i), \quad \forall i \in[1, n], \forall k \in \mathbb{N}_{n},
$$

where $\alpha_{j}(k-1, k)=\alpha_{j+1}(k+1)-\alpha_{1}(k) \alpha_{j}(k-1) ; \alpha_{n+1}(k+1)=0$; for all $k \in \mathbb{N}_{0}$. For any nontrivial solution, there is an integer $i(k) \in[1, n]$, dependent on $k$, such that $\delta(k-i(k), k-i(k))>0$ so that $\delta(k-i, k+1)<0$ if

$$
\alpha_{i(k)}(k-1, k)>-\frac{\sum_{i(k) \neq j=1}^{n} \alpha_{j}(k-1, k) \delta(k-j, k-i(k))}{\delta(k-i(k), k-i(k))}=-\frac{\sum_{i(k) \neq j=1}^{n} \alpha_{j}(k-1, k) x(k-j)}{x(k-i(k))}
$$

and the solution possesses at least one generalized weak zero in $[k-n, k]$. Any solution is GO if (5.11) holds for nonzero initial conditions and all $k \in \mathbb{N}_{n}$. By zeroing $\alpha_{j}(k-1, k)$, $j \in[1, n-1]$, it follows that $x(k+1)=\alpha_{1}(k) \alpha_{n}(k-1) x(k-n)$ and any solution is then GSSO if for all $k \in \mathbb{N}_{n}$

$$
\alpha_{j}(k+1)=\alpha_{1}(k) \alpha_{j}(k-1), \quad j \in[1, n-1], \quad \alpha_{1}(k) \alpha_{n}(k-1)<0 .
$$

Note that both conditions (5.12) together imply that $\alpha_{n}(k)=\alpha_{1}(k) \alpha_{n-1}(k-1), \alpha_{1}(k+$ 1) $\alpha_{n}(k)<0$. Extending the notation $\alpha_{j}(k-\ell, k)$ for any integers $\ell \in \mathbb{N}_{1}, j \in[1, n-\ell]$, $x(k+1)$ might be parameterized as $x(k+1)=-\sum_{j=1}^{n} \alpha_{j}(k-\ell, k) x(k-\ell-j+1)$. Thus, (5.12), guaranteeing the presence of weak generalized zeros, may be generalized as

$$
\alpha_{i(\ell, k)}(k-\ell, k)>-\frac{\sum_{i(\ell, k) \neq j=1}^{n} \alpha_{j}(k-\ell, k) x(k-\ell-j+1)}{x(k-i(\ell, k))} .
$$

\section{Acknowledgment}

The author thanks the Spanish Ministry of Education for its support through Grant DPI 2006-00714.

\section{References}

[1] R. P. Agarwal and S. R. Grace, "Oscillation theorems for certain functional differential equations of higher order," Mathematical and Computer Modelling, vol. 39, no. 9-10, pp. 1185-1194, 2004.

[2] R. P. Agarwal and W.-T. Li, "Interval oscillation criteria for second-order nonlinear perturbed differential equations," Computers \& Mathematics with Applications, vol. 47, no. 4-5, pp. 751$765,2004$. 
[3] S. H. Saker, P. Y. H. Pang, and R. P. Agarwal, "Oscillation theorems for second order nonlinear functional differential equations with damping," Dynamic Systems and Applications, vol. 12, no. 3-4, pp. 307-321, 2003.

[4] B. G. Zhang and R. P. Agarwal, "The oscillation and stability of delay partial difference equations," Computers \& Mathematics with Applications, vol. 45, no. 6-9, pp. 1253-1295, 2003.

[5] R. P. Agarwal, M. Bohner, S. R. Grace, and D. O’Regan, Discrete Oscillation Theory, vol. 1 of Contemporary Mathematics and Its Applications, Hindawi, New York, NY, USA, 2005.

[6] J. C. Soto and M. De la Sen, "Nonlinear oscillations in nonperiodic sampling systems," Electronics Letters, vol. 20, no. 20, pp. 816-818, 1984.

[7] M. De la Sen, "Preserving positive realness through discretization," Positivity, vol. 6, no. 1, pp. 31-45, 2002.

[8] M. De la Sen, "Some conceptual links between dynamic physical systems and operator theory issues concerning energy balances and stability," Informatica, vol. 16, no. 3, pp. 395-406, 2005.

[9] R. P. Agarwal, K. Perera, and D. O’Regan, "Multiple positive solutions of singular problems by variational methods," Proceedings of the American Mathematical Society, vol. 134, no. 3, pp. 817824, 2006.

[10] R. P. Agarwal, H. Lü, and D. O’Regan, "Positive solutions for the singular p-Laplace equation," Houston Journal of Mathematics, vol. 31, no. 4, pp. 1207-1220, 2005.

[11] M. De la Sen, "Absolute stability of feedback systems independent of internal point delays," IEE Proceedings of Control Theory and Applications, vol. 152, no. 5, pp. 567-574, 2005.

[12] M. De la Sen, "Sufficiency-type stability and stabilization criteria for linear time-invariant systems with constant point delays," Acta Applicandae Mathematicae, vol. 83, no. 3, pp. 235-256, 2004.

[13] T. Kaczorek, "Neural networks of positive systems," in Artificial Intelligence and Soft Computing (ICAISC '04), vol. 3070 of Lecture Notes in Artificial Intelligence, pp. 56-63, Springer, Berlin, Germany, 2004.

[14] S. Walczak and D. Idczak, "Positive systems with non decreasing controls. Existence and wellposedness," in Positive Systems (Rome, 2003), vol. 294 of Lecture Notes in Control and Information Sciences, pp. 369-376, Springer, Berlin, Germany, 2003.

M. De la Sen: Department of Electricity and Electronics, Faculty of Science and Technology, University of the Basque Country, Campus of Leioa, Bizkaia, Aptdo, 544 Bilbao, Spain Email address: manuel.delasen@ehu.es 


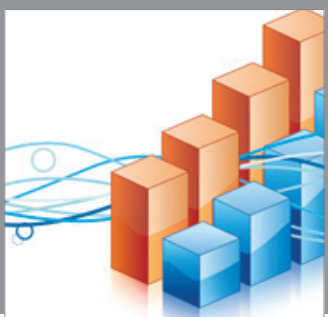

Advances in

Operations Research

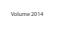

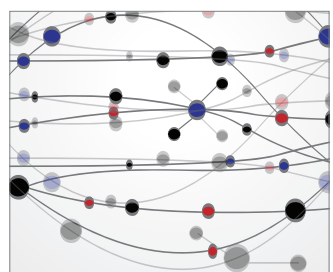

\section{The Scientific} World Journal
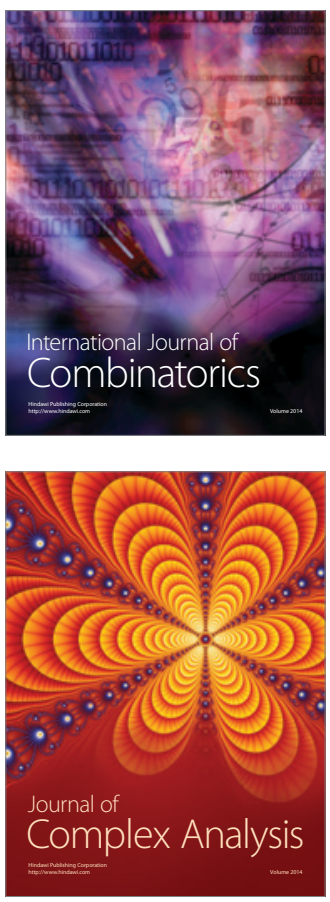

International Journal of

Mathematics and

Mathematical

Sciences
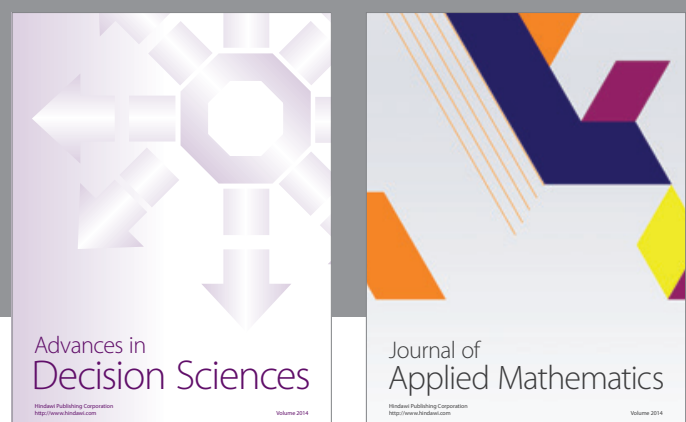

Journal of

Applied Mathematics
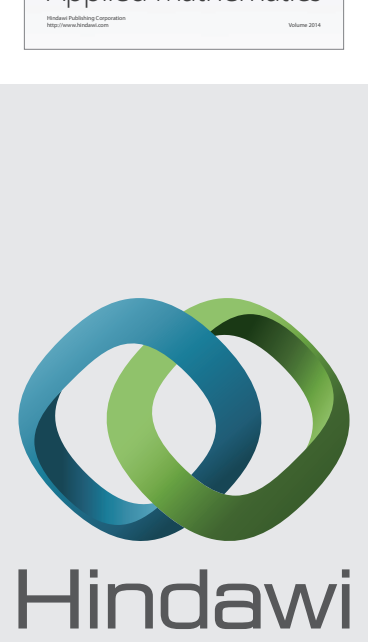

Submit your manuscripts at http://www.hindawi.com
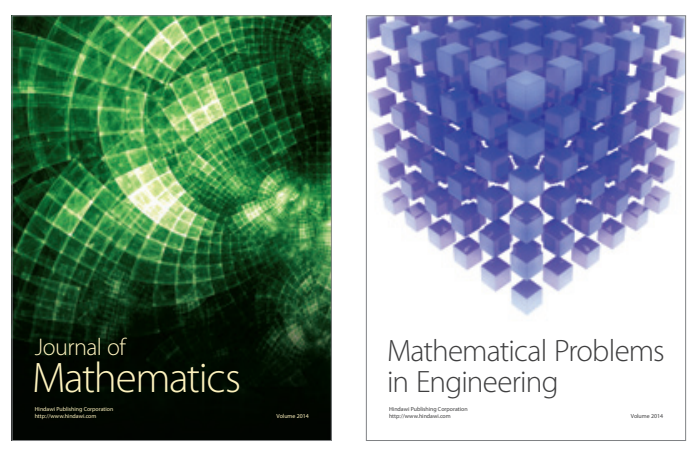

Mathematical Problems in Engineering
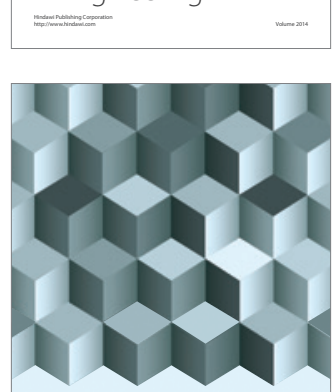

Journal of

Function Spaces
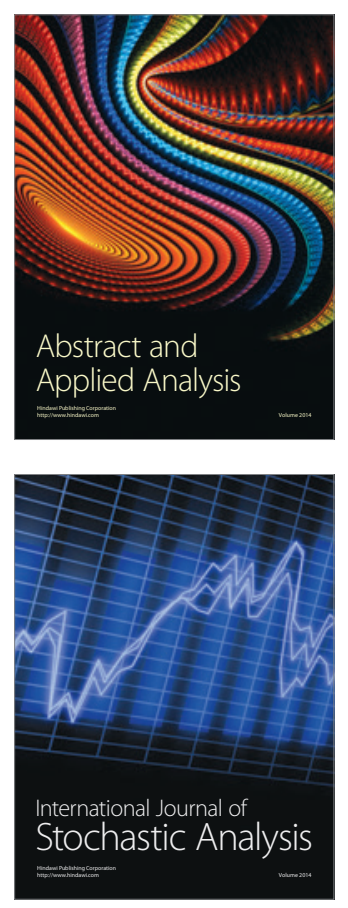

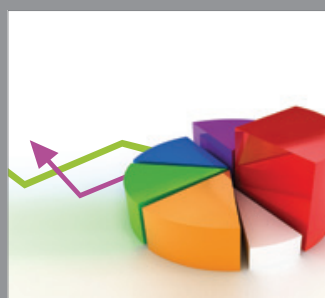

ournal of

Probability and Statistics

Promensencen
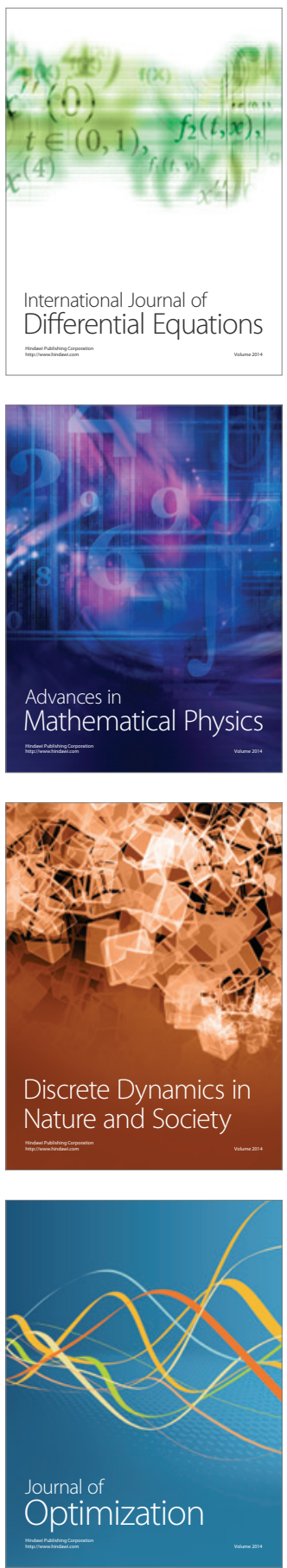